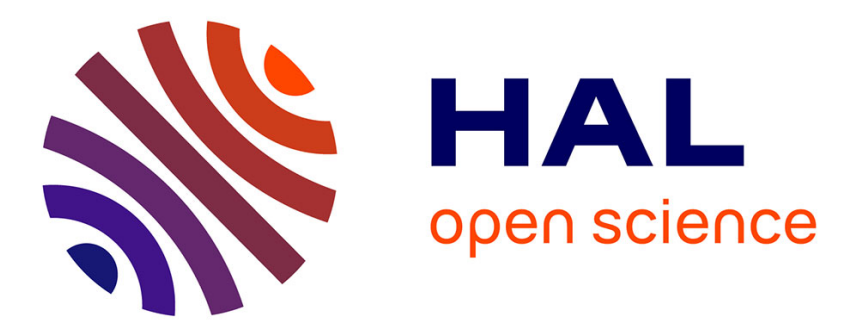

\title{
Quelques aspects récents du traitement optique des images
}

D. Charraut, J. Duvernoy, J.M. Fournier, J.P. Goedgebuer, A. Lacourt, G. Tribillon, J. Ch. Viénot

\section{- To cite this version:}

D. Charraut, J. Duvernoy, J.M. Fournier, J.P. Goedgebuer, A. Lacourt, et al.. Quelques aspects récents du traitement optique des images. Revue de Physique Appliquée, 1976, 11 (2), pp.227-236. 10.1051/rphysap:01976001102022700 . jpa-00244052

\section{HAL Id: jpa-00244052 https://hal.science/jpa-00244052}

Submitted on 1 Jan 1976

HAL is a multi-disciplinary open access archive for the deposit and dissemination of scientific research documents, whether they are published or not. The documents may come from teaching and research institutions in France or abroad, or from public or private research centers.
L'archive ouverte pluridisciplinaire HAL, est destinée au dépôt et à la diffusion de documents scientifiques de niveau recherche, publiés ou non, émanant des établissements d'enseignement et de recherche français ou étrangers, des laboratoires publics ou privés. 


\title{
QUELQUES ASPECTS RÉCENTS DU TRAITEMENT OPTIQUE DES IMAGES
}

\author{
D. CHARRAUT, J. DUVERNOY, J. M. FOURNIER, J. P. GOEDGEBUER, \\ A. LACOURT, G. TRIBILLON et J. Ch. VIÉNOT \\ Laboratoire de Physique Générale et Optique (*) \\ (Holographie et traitement optique des signaux), 25030 Besançon, Cedex, France
}

Introduction. - Les techniques de l'holographie et du filtrage adapté semblaient avoir, il y a quelques années, fixé les modalités de traitement optique de l'information dans des corrélateurs plus ou moins sophistiqués, où un faisceau monochromatique cohérent jouait le rôle de porteuse. La mise en œuvre des propriétés spatio-temporelles de faisceaux polychromatiques et des effets de speckle d'une part, la nécessité de considérer des données dont la formation ou l'acquisition font appel à des processus non nécessairement linéaires d'autre part, ont conduit à repenser le problème du traitement optique et à envisager des modèles peu familiers pour l'opticien.

On présente deux groupes de méthodes développées dans des domaines d'application assez différents. Le premier a trait à l'analyse de signaux bidimensionnels représentant un codage graphique de l'information, le second à la détermination des états de surface.

La première approche concerne les méthodes de couplage entre l'optique - au sens d'une technique analogique - et des méthodes de calcul — au sens d'une technique digitale. On peut faire d'entrée une distinction entre traitement optique de l'information et traitement de l'information optique. La situation en traitement optique de l'information, axée essentiellement sur les problèmes de reconnaissance des formes, amène à de nombreux obstacles (difficultés de construction, voire de conception des interfaces pour l'entrée et la sortie des données, qui se posent en termes de matériaux, de capteurs et de conversion numérique des résultats fournis sous forme analogique). Pourtant l'un des intérêts du dispositif optique réside dans la grande quantité d'informations qui peut être analysée en une seule opération. De plus son adaptation directe au traitement des images est incontestable. Ceci suggère l'utilisation de méthodes hybrides.

Par traitement de l'information optique on entendra un ensemble de processus plus larges. Ici le système optique élague d'abord l'information, la fait sortir d'images (au sens large) qui ne sont pas directement

(*) Associé au C. N. R. S. digitalisables. Alors l'utilisation de données, sous forme élaborée, permet, par un traitement purement optique (par exemple le profil des pics de corrélation), la comparaison entre un signal de référence et un ensemble de signaux de même nature ou d'une même classe d'objets : ainsi la figure 1 illustre-telle une étapefinale directement accessible optiquement sur l'étude de la ressemblance moyenne $R$ entre des textes écrits par plusieurs scribes.

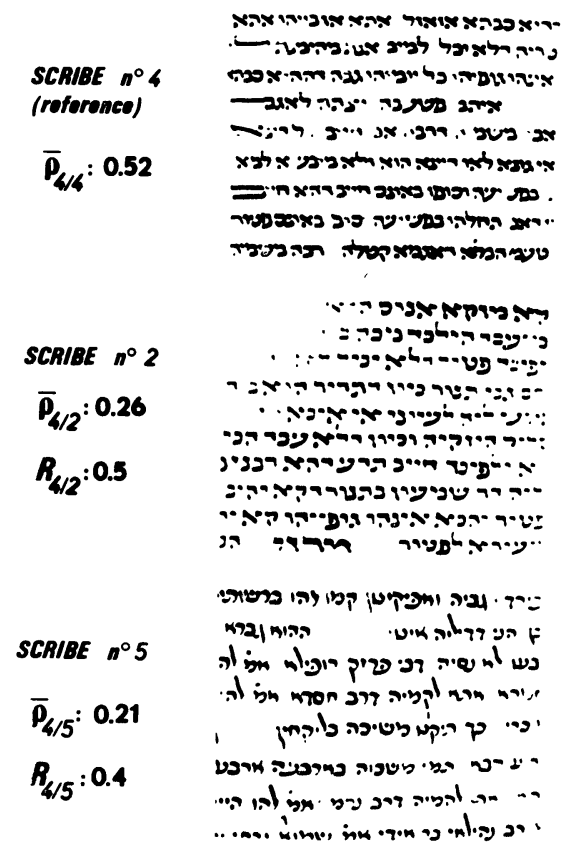

Fig. 1. - Evaluation de la ressemblance $\mathbf{R}$ entre les textes écrits par le scribe $n^{\circ} 2$ et le scribe $n^{\circ} 4$ d'une part, le scribe $n^{\circ} 5$ et le scribe $n^{\circ} 4$ d'autre part. $\rho$ représente la ressemblance moyenne lettre à lettre entre les échantillons écrits par le scribe $n^{\circ} 4$ et ceux des autres textes.

On sait que l'opticien dispose de moyens permettant une économie de temps de traitement, précisément par l'élagage a priori des données, de moyens de réduction d'encombrement de la mémoire centrale au niveau de 
laquelle interviennent les opérations complexes de corrélation, de convolution, de réalisation de moyennes, de modification des formes au niveau des spectres ou des images.

Une approche pas à pas peut être schématisée :

1) D'abord suivant un aspect statistique de l'information contenue dans le spectre de Fourier. Ainsi la comparaison d'un cliché original du Palais de Chaillot et le même cliché filtré (Fig. 2) fait ressortir deux
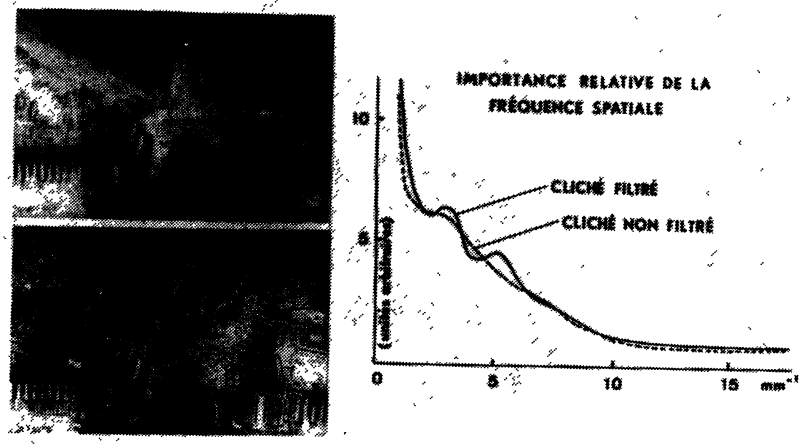

FIG. 2. - Explication dans le texte.

« accidents » sur la courbe du cliché filtré, figurant deux bandes de fréquences que l'on peut considérer comme caractéristiques de la meilleure visibilité de l'image. Les figures 3 , 4 et 5 précisent cette notion de bande caractéristique.

2) En introduisant le concept de facteur de forme: si l'on dispose d'une collection de signaux de même nature, on visualise une donnée statistique caractéristique par exemple de la forme moyenne des signaux (Fig. 6). Une caractéristique plus générale peut être définie lorsqu'on s'intéresse à la genèse ou encore à la

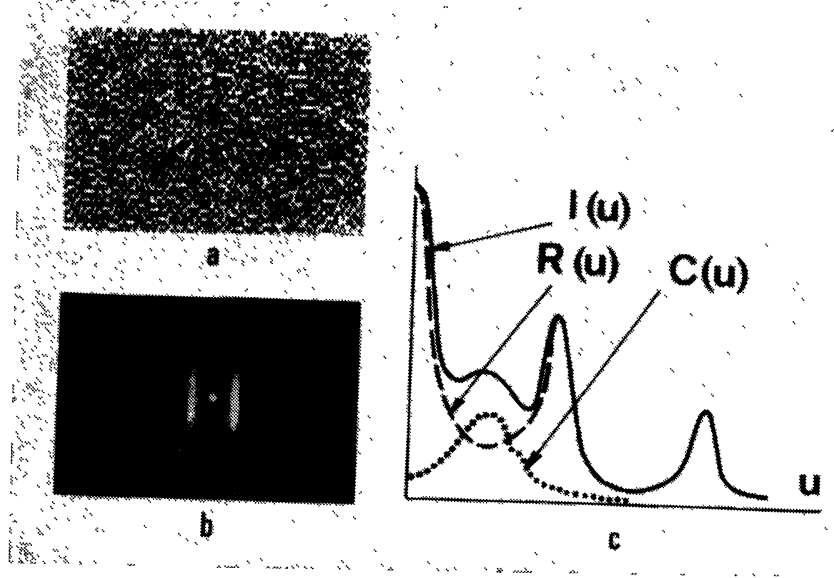

FIG. 3. - Bande caractéristique $\mathrm{C}(u)$ et redondance $\mathrm{R}(u)$ dans le spectre $\mathrm{I}(u)$ de la grille pseudo-aléatoire $(a)$ dont la figure de diffraction à l'infini est reproduite en $(b)$. l. inregivtrement dimages es unc operation courante (a) interessant plus partice graphic et linstrumentation reproduction dinformations deul niscaus de luminance. mation proprement dite et De tels yntemes, appeles

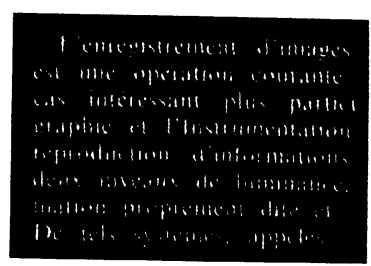

FIG. 4. - Le texte de gauche étant codé par la bande limitée $C(u)$ (enregistrement holographique où la grille précédente est utilisée comme source de référence) la restitution (à droite) est encore lisible.

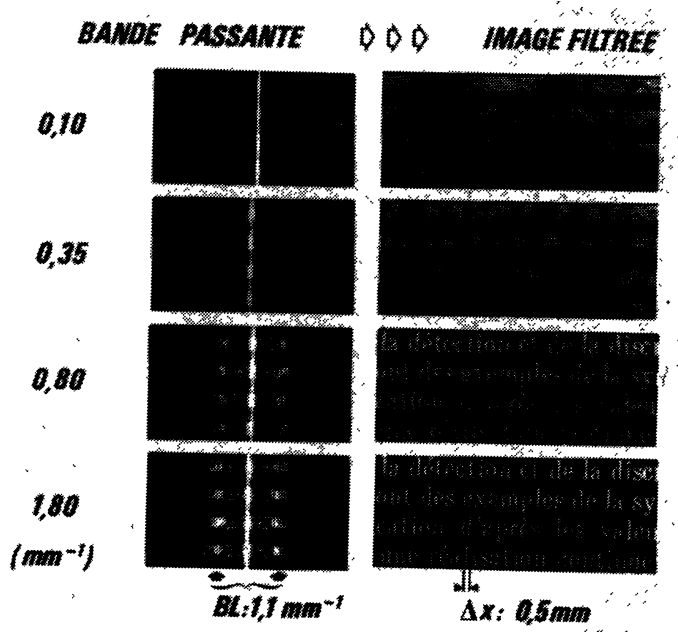

Fig. 5. - Influence de la largeur de bande passante sur la qualité de l'image restituée. La lisibilité des lettres est atteinte pour une largeur de bande passante supérieure à $0,35 \mathrm{~mm}^{-1}$.

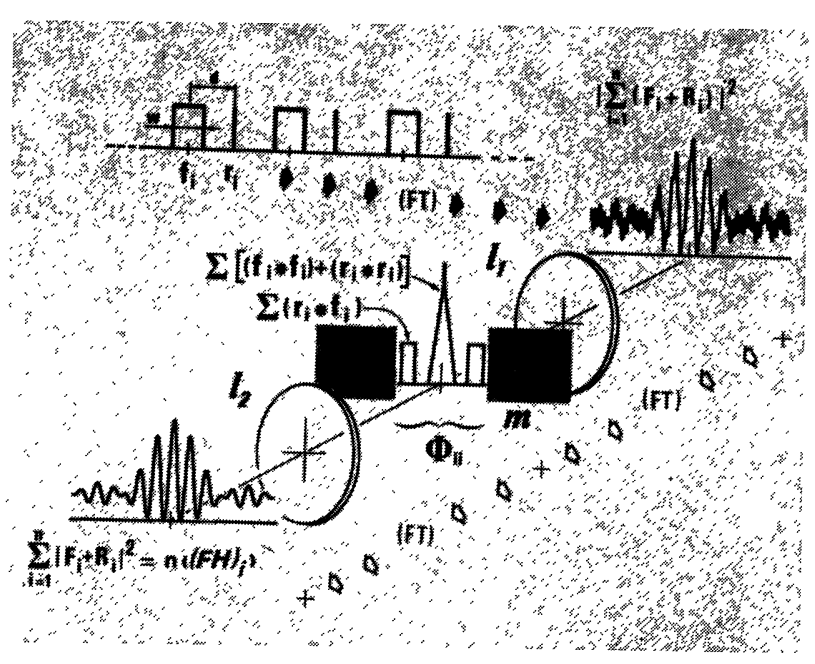

FIG. 6. - L'hologramme d'une distribution de signaux tels que $f_{i}$ (peu différents les uns des autres) est décrit par $\left|\Sigma\left(F_{i}+R_{i}\right)\right|^{2}$ $\left(r_{i}\right.$ est l'impulsion de référence associée à $f_{i}$ ). Il est placé à l'entrée d'un corrélateur $\left(l_{1}, l_{2}\right)$. Dans la région centrale du plan de Fourier le terme $\varphi_{i i}$ comprend les autocorrélations de $f_{i}$ et de $r_{i}$ et les intercorrélations $\Sigma r_{i} * f_{i}$ et $\Sigma f_{i} * r_{i}$ représentant les signaux moyens $\left\langle f_{i}\right\rangle$ puisque $r_{i}$ est une mesure de Dirac. A la sortie du corrélateur on obtient l'hologramme de Fourier moyen $<\mathrm{FH}_{i}>$ lorsque l'on a isolé $\varphi_{i i}$ au moyen du masque $\mathrm{M}$. 
génération d'une classe d'objets de même nature. Il s'agit d'un opérateur qui crée les différents éléments de la classe à partir d'un prototype. On obtient différents types d'écriture suivant la forme mise en mémoire dans l'hologramme (Fig. 7). Le facteur de forme est la

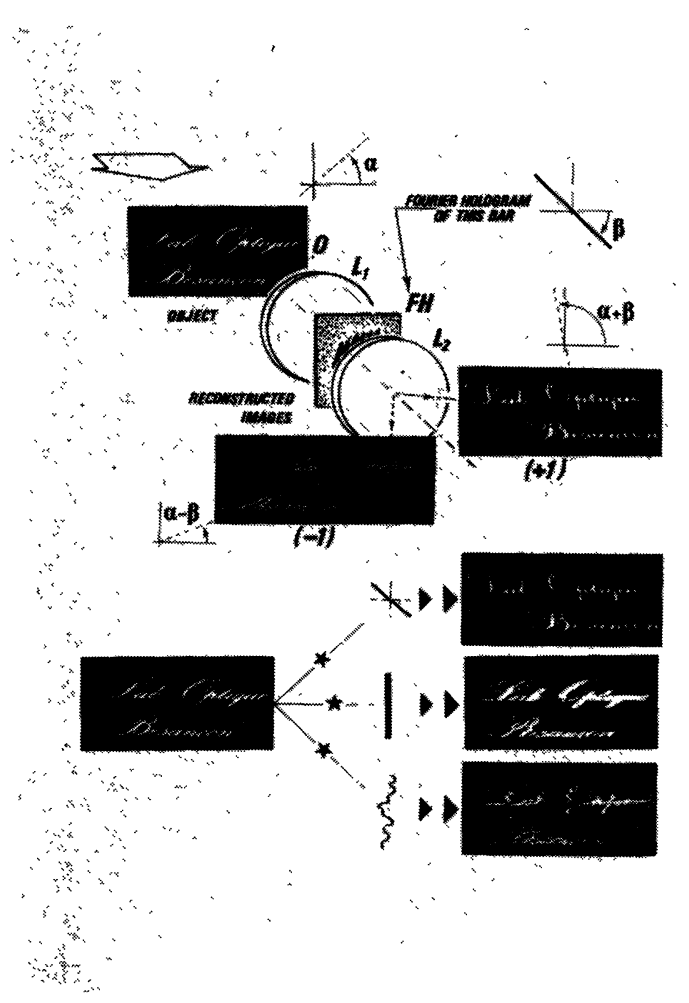

Fig. 7. - Dans le corrélateur $\left(\mathrm{L}_{1}, \mathrm{~L}_{2}\right)$ à l'entrée duquel se trouve le message objet, l'hologramme de Fourier FH est celui d'une barre inclinée (en haut), d'un trait épais (au centre) ou d'une ligne sinueuse (en bas). Le résultat de l'opération se traduit dans les images \pm 1 dans le plan de sortie.

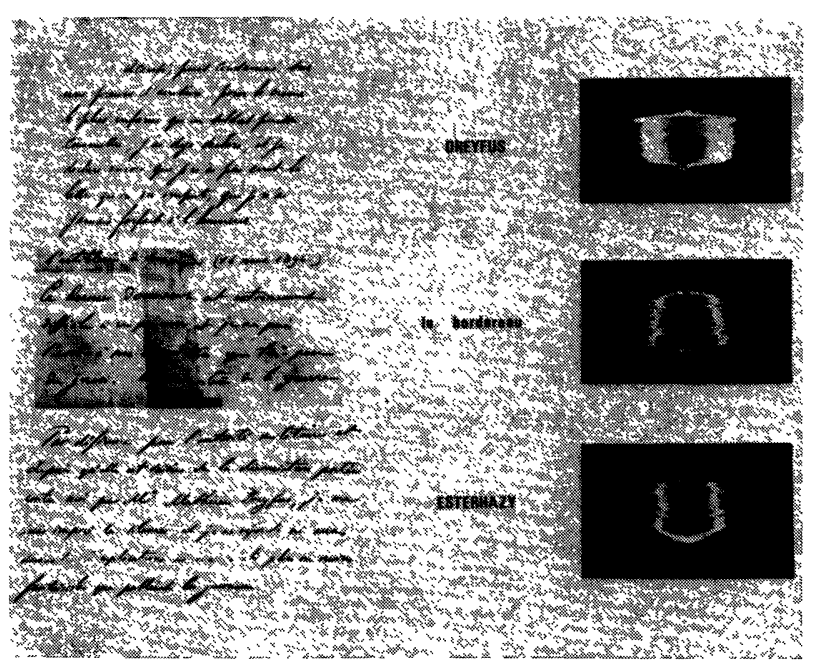

Fig. 8. - Une représentation des facteurs de forme (à droite) correspondant à 3 échantillons d'écriture (à gauche) montre l'incompatibilité entre le bordereau et un échantillon de l'écriture de Dreyfus. fonction d'appareil qui permet de passer de ce qu'on appelle l'objet dans cette figure à l'image effective. Par exemple il décrit l'effet de la main du scribe dans un texte manuscrit. Une application de la mise en évidence des facteurs de forme est donnée dans l'expertise du bordereau de l'affaire Dreyfus (Fig. 8).

Une approche statistique plus puissante nécessite l'introduction de modèles faisant appel à un échantillonnage dans le spectre du message à traiter, puis à une transformation mathématique dont il sera question plus loin. On aboutit alors à une modélisation mathématique. On montre que le transfert effectif entre le prototype et le résultat de l'action des différents opérateurs est en général non linéaire (Fig. 9).

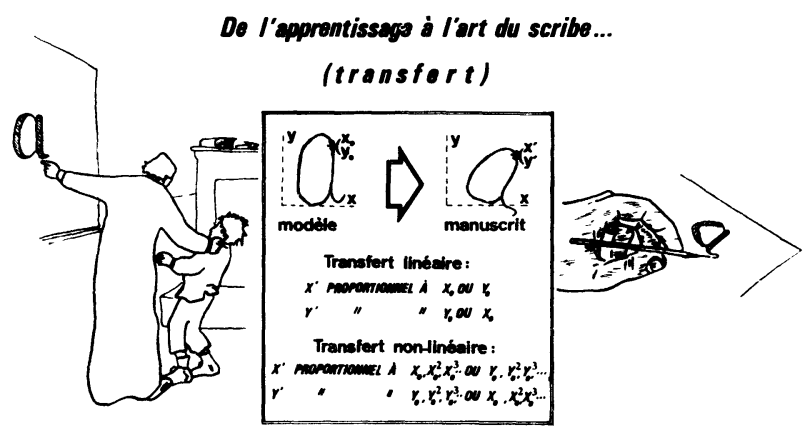

FIG. 9.

1. Procédures mixtes, analogiques et digitales. Le traitement optique de l'information s'adresse à des catégories d'objets dont la forme est susceptible de varier, reconnaître un objet c'est d'abord déterminer à quelle catégorie il appartient, puis c'est ensuite donner ses caractéristiques propres à l'intérieur de sa catégorie. La reconnaissance des formes suppose donc un apprentissage conduisant à une description statistique des classes d'objets en présence. L'identification de la forme résulte alors d'une procédure de décision basée sur la mise en œuvre de fonctions mathématiques appropriées.

Au niveau de l'acquisition des données on dispose de capteurs et de dispositifs de traitement optiques capables d'effectuer des opérations complexes sur des objets représentant de grandes quantités d'information - tels que les images. Cependant les opérations disponibles - par exemple : la corrélation, la multiplication ou la soustraction d'images - sont réalisées de façon analogique. Comme le traitement optique de l'information ne dispose pas directement d'opérations logiques, il faut réaliser une conversion numérique des données extraites afin de permettre leur exploitation statistique.

Il est inutile de revenir sur la puissance des méthodes mathématiques de reconnaissance des formes qui s'exprime essentiellement à travers le calcul numérique. La position du traitement optique n'est pas dans la 
concurrence avec l'ordinateur. Il trouve une place originale en amont, au niveau de la mise en forme et de l'extraction des données caractéristiques.

Le thème développé ici concerne l'utilisation de modèles optiques ou de traitements optiques pour la mise en forme des données. Celle-ci peut être soit directe : dans ce cas des mesures physiques sont effectuées - soit indirecte : dans ce cas c'est un modèle d'imagerie optique qui définit les variables à prendre en considération.

Ces deux cas se distinguent par la nature du signal à traiter. Ils seront illustrés ici sur l'exemple de l'écriture considérée comme un processus d'imagerie optique : en effet on peut dire que les lettres écrites par un individu à l'instant $t$ sont les images de celles qu'il a apprises à l'instant $t_{0}$. Le scripteur est équivalent à un système optique, linéaire ou non selon que son écriture est régulière ou non.

Caractériser une donnée optique c'est d'abord en fournir une représentation mathématique satisfaisante. De la façon la plus classique et dans le but de faciliter le traitement sur ordinateur, on cherche une expression analytique dans une base orthonormée. La mise en évidence des classes d'objets et leur reconnaissance s'effectuent en considérant les variations statistiques affectant les composantes des objets dans la base choisie. La démarche est schématisée figure 10 : deux descriptions mathématiques consistantes peuvent être données, suivant la nature du signal optique considéré.

\begin{tabular}{|c|c|c|c|c|}
\hline $\begin{array}{c}\text { LINEAR PAOCESS } \\
\text { FOURIER TRAMSF.' } \\
c_{n} \\
n=1, \cdots, n\end{array}$ & $\begin{array}{c}C_{1} \\
\vdots \\
\vdots \\
\vdots \\
C_{N}\end{array}$ & 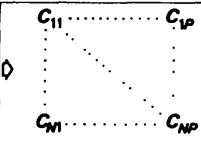 & \begin{tabular}{|c|}
$K$ \\
$a$ \\
$h$ \\
$u$ \\
$n$ \\
$o$ \\
$n$ \\
\end{tabular} & $\begin{array}{l}\left(\boldsymbol{\Lambda}_{1}\right) \\
\left(\mathbf{\Lambda}_{2}\right) \\
\nearrow\end{array}$ \\
\hline $\begin{array}{c}\text { NON LINEAR } \\
\text { PROCESS } \\
\text { PARAM. TRAMSF.' } \\
\kappa_{m} \\
m=1, \cdots, m\end{array}$ & $\begin{array}{c}K_{1} \\
\vdots \\
\vdots \\
\vdots \\
K_{m}\end{array}$ & 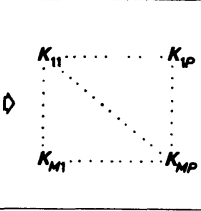 & 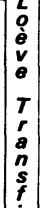 & $\frac{i f, \Lambda_{1}, \Lambda_{2} \gg \Lambda_{j}, \ldots}{k l_{1}, k l_{2}}$ \\
\hline $\begin{array}{c}\text { spatiol } \\
\text { description } \\
\text { (simese sismal) }\end{array}$ & & $\begin{array}{l}\text { statistical } \\
\text { description } \\
\text { (P signns) }\end{array}$ & & $\begin{array}{l}\text { eigenvectors } \\
\text { description } \\
\text { (ITR:SIC VARLABES) }\end{array}$ \\
\hline
\end{tabular}

FIg. 10. - Schématisation des processus linéaires et non linéaires.

Lorsque le signal est fourni par un processus linéaire - ou bien la représente - il est entièrement défini par ses composantes de Fourier $C_{n}$. C'est le cas de l'imagerie optique et des écritures régulières. Lorsque le signal ne résulte pas d'un processus linéaire, on peut en donner une description paramétrique où des coefficients $K_{m}$ jouent le rôle de composantes. Dans les deux cas les bases sont orthonormales.

Considérons maintenant une classe de $P$ signaux : les composantes $C_{n}$ et $K_{m}$ montrent des variations statistiques. Ces variations affectent simultanément l'ensemble des composantes, ce qui rend difficile la caractérisation des classes de signaux en présence. On voit donc qu'une transformation de coordonnées est nécessaire : en effet une matrice de changement de base redistribue sur de nouveaux axes les variations qui se produisent sur l'ensemble des anciens axes. Une transformation adaptée à l'analyse statistique doit concentrer ces variations sur un petit nombre d'axes caractéristiques : on extrait ainsi les paramètres déterminants. C'est le cas de la transformation de Karhunen-Loève : le nouveau système d'axes est constitué par les vecteurs propres $k l_{i}$ de la matrice de covariance des signaux dans la base $C_{n}$ ou $K_{m}$. Watanabe a démontré que les valeurs propres $\Lambda_{i}$ sont reliées à la quantité d'information transportée par les vecteurs propres $k l_{i}$ pour la description des signaux.

Supposons qu'un petit nombre de valeurs propres, deux par exemple, soient dominantes. Une telle condition montre que les deux premiers vecteurs propres transportent pratiquement toute l'information. Dans ce cas les signaux dépendent pratiquement de deux variables : leur dimension intrinsèque est égale à $2 . \mathrm{Si}$ une telle condition n'est pas réalisée, on peut dire que la dimension du phénomène reste $N$ ou $M$. Il n'y a pas de variables caractéristiques. Les applications présentées illustrent les deux types de signaux envisagés ici.

Prenons l'exemple d'une écriture régulière (Fig. 11). Les lettres manuscrites sont les images des lettres

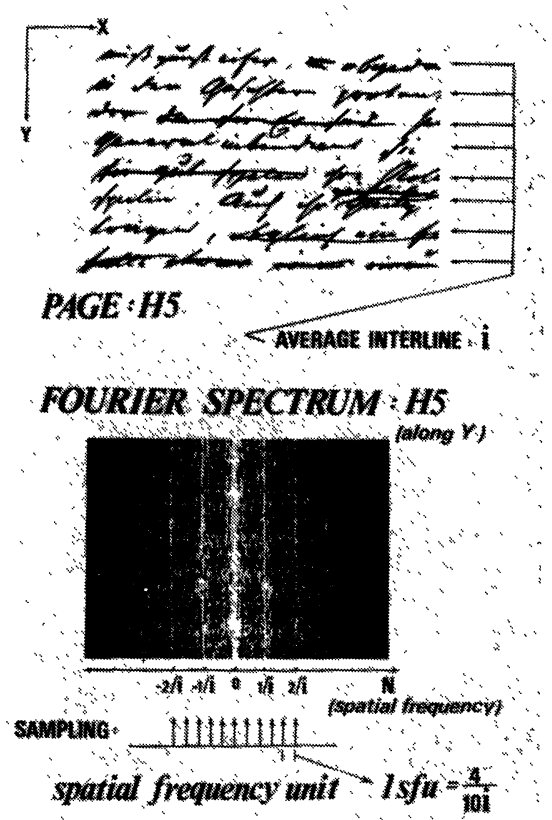

FIG. 11. - Echantillonnage dans le spectre de Fourier d'une page de texte.

apprises par le scripteur. Ici on considérera que le système optique équivalent ne présente pas d'aberrations et sa fonction de transfert est une caractéristique $\mathrm{du}$ scripteur. Cette fonction est accessible dans le 
spectre de Fourier de la page manuscrite. Un échantillonnage de ce type donne les composantes $C_{n}$ définissant le transfert du scripteur pour la page considérée.

Cet échantillonnage a été effectué sur les spectres de Fourier de 16 pages écrites par Henri Heine et 5 pages écrites par ses secrétaires. En prenant les deux premiers vecteurs propres $k l_{1}$ et $k l_{2}$ on conserve $98 \%$ de l'information relative aux variations du transfert dans l'ensemble des pages. Une représentation des pages dans le plan $\left(k l_{1}, k l_{2}\right)$ montre l'existence de deux domaines séparés (Fig. 12), l'un englobant les pages de

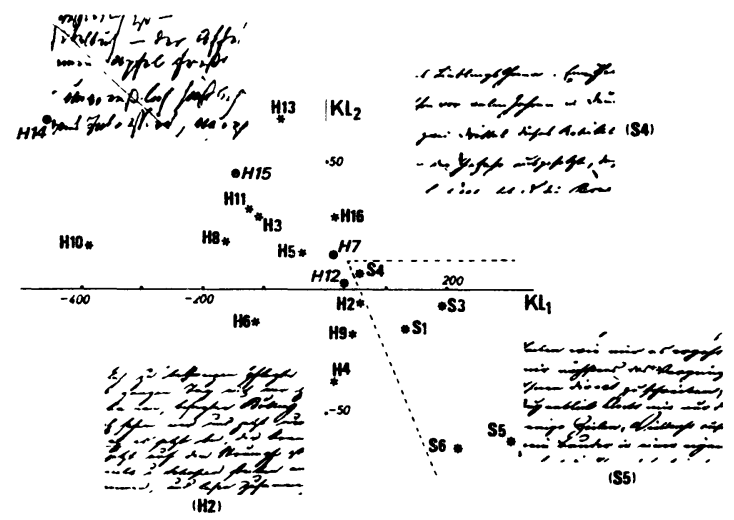

Fig. 12. - Explication dans le texte.

Heine, notées $H_{i}$, l'autre celles des secrétaires, notées $S_{i}$, Dans ce cas la séparation en classe ne semble pas poser de problème. L'étude de la structure interne et de l'évolution d'une classe seule est plus difficile. Ainsi par exemple la matrice de covariance du seul groupe de 16 pages écrites par Henri Heine entre 1820 et 1855. montre qu'il faut prendre ses trois premiers vecteurs propres, $k l_{1}, k l_{2}$ et $k l_{3}$ pour retenir $98 \%$ de l'information. La représentation correspondante des pages est donnée figure 13. En faisant l'hypothèse d'une évolu-

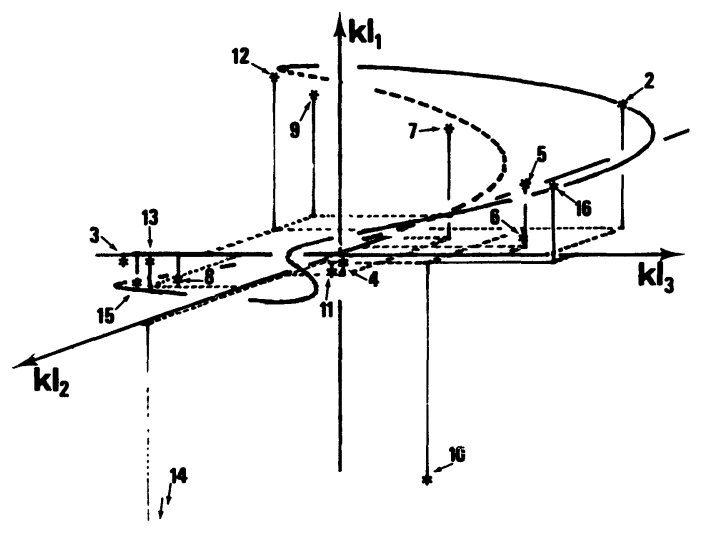

FIG. 13. - Représentation tridimensionnelle des pages de texte dans l'espace des trois vecteurs propres principaux. La courbe d'évolution a été ajustée pour passer au plus près de l'ensemble des points. tion continue de l'écriture, c'est-à-dire d'une variation du transfert en fonction du temps, il faut trouver une courbe unique passant par l'ensemble des points. Le long de cette courbe les distances entre points successifs seront mesurées en abscisse curviligne $S$. Les distances temporelles sont données en fonction du temps $t$. Il faut alors établir une relation entre $S$ et $t$ (Fig. 14). Ces résultats correspondent à des écritures

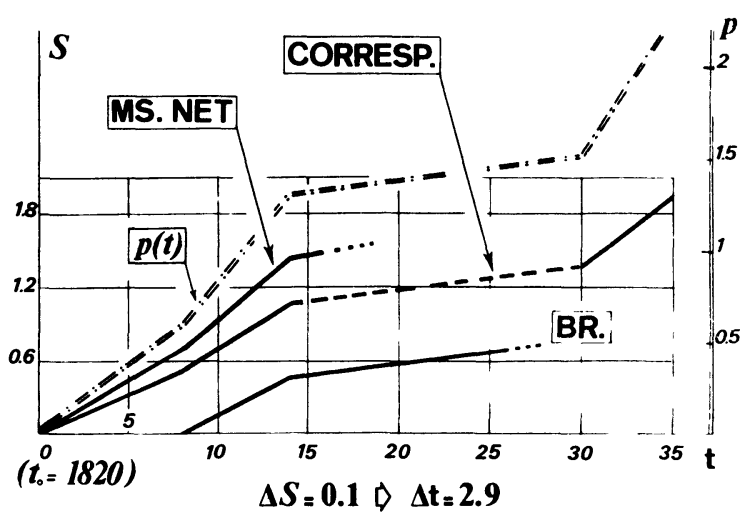

FIG. 14. - Sous réserve de distinguer 3 types d'écritures : brouillon, mise au net et correspondance, il existe une relation caractéristique entre le temps $t$ et l'abscisse curviligne $S$. Une telle abaque indique qu'il serait possible de dater une page avec une précision de 3 ans sur un intervalle de 35 ans. Le nombre d'échantillons sur lesquels on a travaillé étant relativement réduit on ne saurait donner des conclusions définitives. Des travaux en cours reprennent cette méthode à partir de centaines de pages.

régulières. Dans le cas de tracés irréguliers on ne peut pas définir un système optique parfait équivalent au scripteur. Il faut utiliser un modèle d'imagerie aberrante (Fig. 15).

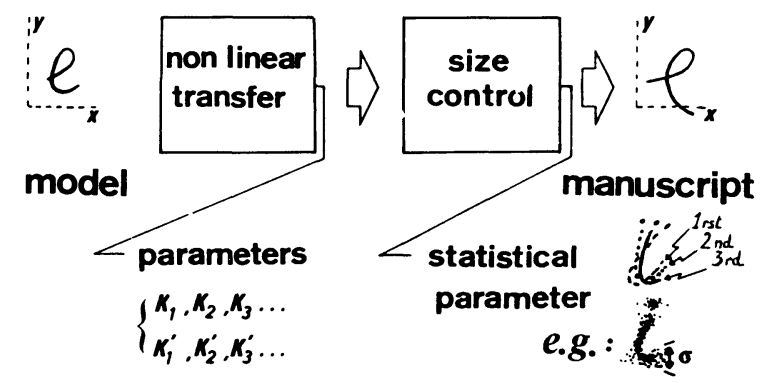

FIG. 15. - Les paramètres représentés à gauche décrivent des tendances dans le transfert non linéaire. Une pondération de ces paramètres (à droite) conduit au tracé effectif.

Considérons par exemple une lettre et son image aberrante : la lettre manuscrite. En appliquant une transformation ponctuelle sur les coordonnées de l'objet il est possible de synthétiser le tracé de la lettre manuscrite. Les coordonnées de cette lettre sont don- 
nées au moyen de polynômes orthogonaux fonction des coordonnées de l'objet. Les coefficients $K_{m}$ de ces polynômes sont déterminés empiriquement pour ajuster le tracé.

L'influence de ces coefficients est illustrée figure 16.

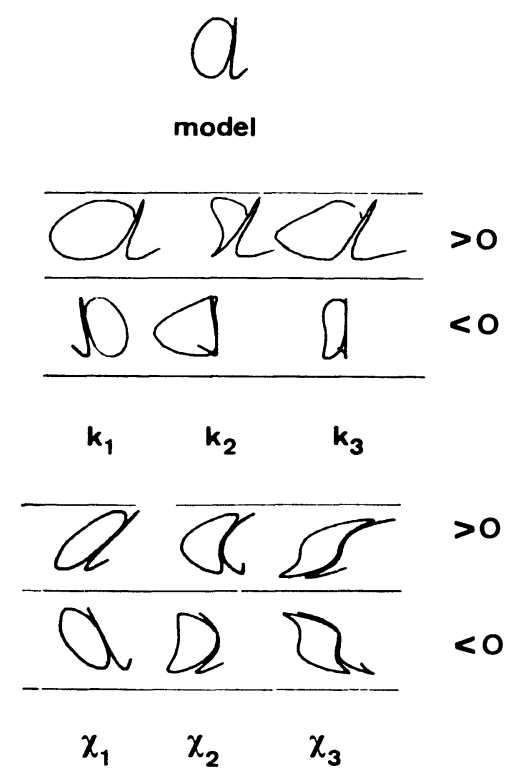

Fig. 16. - Partant d'un modèle de lettre on synthétise différents tracés à l'aide de polynômes de degré croissant. Les deux premières lignes montrent l'influence de réponses non linéaires sur la coordonnée $x$, c'est-à-dire un déplacement des points le long de l'axe $x$ proportionnel aux puissances 1,2 et 3 de $x$ (coefficients $K_{1}, K_{2}$ et $K_{3}$ ). Les deux lignes du bas montrent l'influence de couplages non linéaires entre $x$ et $y$, c'est-à-dire un déplacement des points le long de $x$ proportionnel aux puissances 1,2 ou 3 de $y$ (coefficients $\chi_{1}, \chi_{2}$ et $\chi_{3}$ ).

L'ensemble de lettres manuscrites présenté figure 17 a été synthétisé à partir d'un modèle de lettre arbitraire. Chacune est décrite dans la base des coefficients $K_{m}$. La matrice de covariance présente deux vecteurs

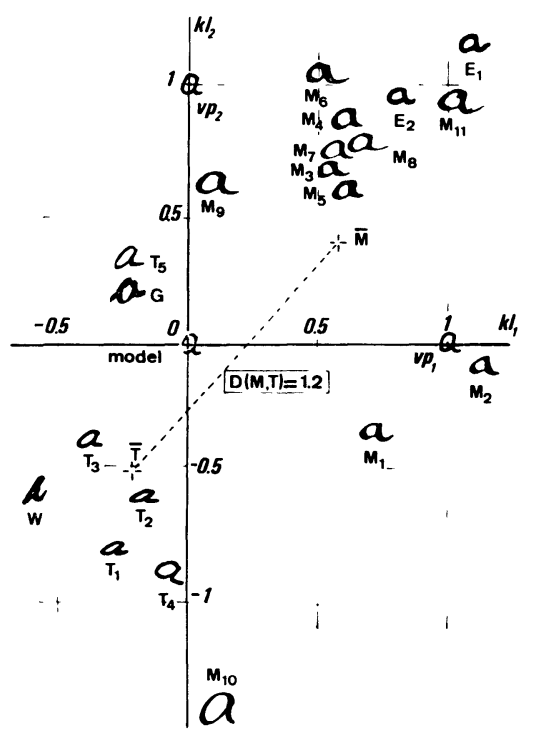

Fig. 17. - Explication dans le texte. propres $k l_{1}$ et $k l_{2}$ dominants. Une représentation des lettres dans le plan de ces deux vecteurs montre clairement la séparation en deux classes : les lettres $a_{\mathrm{M}}$, d'une écriture attribuée à Molière et les lettres $a_{\mathrm{T}}$, d'une écriture témoin. Les lettres $a_{E_{1}}, a_{E_{2}}, a_{\mathrm{W}}$ et $a_{\mathrm{G}}$ ont été synthétisées et projetées dans ce plan après mise en évidence des deux classes ; $a_{E_{1}}$ et $a_{E_{2}}$ étaient identiques à celles effectivement écrites par Molière.

Ainsi semble-t-il nécessaire de doter le traitement optique de l'information d'une dimension statistique en lui associant des techniques d'analyse mathématique sur ordinateur. Réciproquement le traitement optique permet à l'ordinateur de travailler sur des données qui pouvaient lui échapper, ne serait-ce que par la difficulté de digitaliser de grandes quantités d'information.

2. Traitement de l'information optique appliqué à un problème de mécanique. - La connaissance des états de surface est très importante en mécanique de précision. La rugosité, traduisant les fluctuations de niveau autour d'un profil moyen, représente un paramètre essentiel. L'information mise à la disposition de l'utilisateur par une valeur chiffrée de la rugosité est le plus souvent incomplète. Aussi paraît-il intéressant de présenter deux méthodes complémentaires fournissant d'une part la rugosité moyenne de la surface à partir d'abaques, d'autre part une visualisation du profil de cette surface suivant des sections transversales. Les deux font intervenir le caractère à la fois spatial et chromatique de la lumière.

\subsection{DÉTERMINATION DE L'ÉCART QUADRATIQUE} MOYEN D'UNE SURFACE RUGUEUSE (RUGOSITÉ MOYENNE). - Une surface rugueuse éclairée en lumière monochromatique donne lieu à un phénomène de speckle. La lumière diffusée contient l'information relative à la surface éclairée. On sait par exemple que la dimension moyenne des grains de speckle définie par ses paramètres statistiques renseigne sur les dimensions de l'objet. Mais'à ce stade, il n'est pas possible de caractériser et encore moins de mesurer la rugosité de la surface éclairée. Cette mesure nécessite une relation supplémentaire susceptible d'être mise en évidence en éclairant la surface par une deuxième longueur d'onde donnant lieu à un deuxième champ de speckle. La comparaison de ces deux speckles en termes de ressemblance doit fournir des renseignements sur la microstructure de l'objet. La ressemblance entre les speckles se traduit par l'existence d'un degré de corrélation qui, dans ce cas, est une fonction de l'écart spectral $\Delta \lambda$ entre les deux longueurs d'onde et de la rugosité moyenne de la surface représentée par l'écart quadratique moyen en profondeur $\sigma_{z}$ si le profil est gaussien, c'est-à-dire si les irrégularités en profondeur sont régies par une loi de densité gaussienne.

Le principe de la méthode est schématisé sur la figure 18. Elle consiste d'abord en un enregistrement photographique des speckles puis en une analyse 

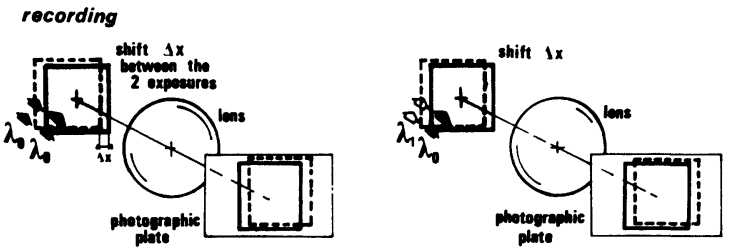

Fourier analysis

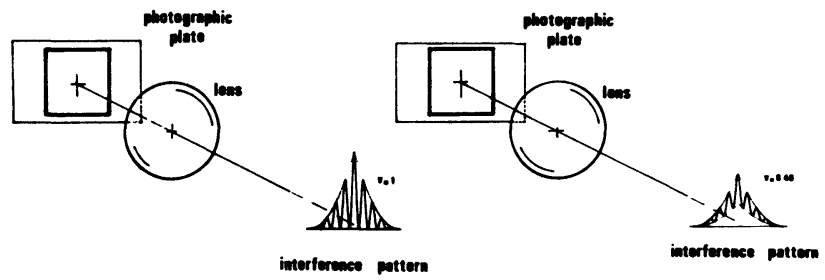

FiG. 18. - Enregistrement et analyse de l'ensemble des images translatées comme indiqué dans le texte.

spectrale de la répartition d'intensité obtenue. A l'image photographique de la surface à étudier on superpose une seconde image, l'objet ayant subi une translation dans son plan de quelques centaines de microns entre les deux expositions. Le film est développé. On effectue alors son analyse spectrale par diffraction d'une onde plane cohérente. L'observation dans le plan focal de la lentille $\mathrm{L}$ donne lieu à un phénomène d'interférence de visibilité 1 où la période des franges est inversement proportionnelle au déplacement effectué entre les deux expositions.

Reprenons le même dispositif, mais modifions la longueur d'onde entre les deux expositions. On observe encore par diffraction un phénomène d'interférence dont la visibilité est inférieure à l'unité. On montre que la visibilité des franges est proportionnelle au degré de corrélation entre les speckles : c'est une fonction de $\Delta \lambda$ et $\sigma_{z}$. La détermination de la rugosité moyenne de la surface se ramène donc à une mesure interférométrique.

La figure 19 rassemble les figures d'interférence observées pour trois états de surface et pour différents

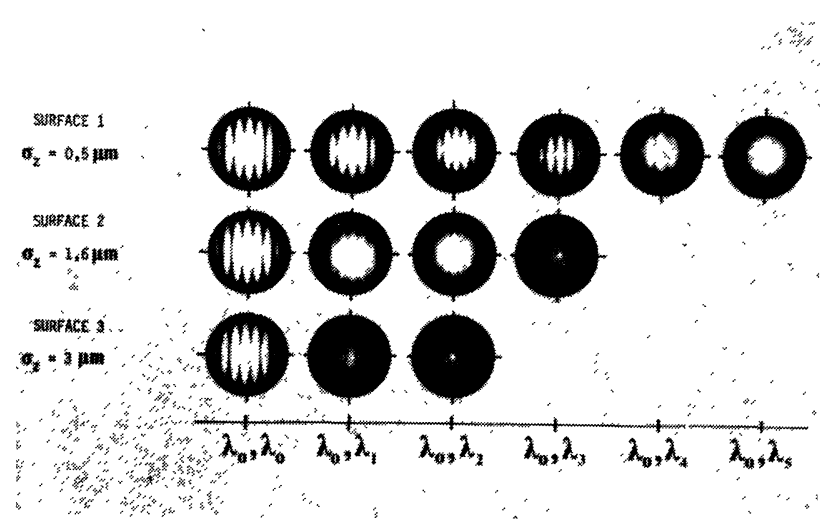

FIG. 19.

$\Delta \lambda$. On note la dépendance de la visibilité des franges avec la rugosité moyenne $\sigma_{z}$ et l'écart spectral $\Delta \lambda$. Le relevé de cette visibilité conduit à une mesure directe de la rugosité moyenne à partir d'une seule figure d'interférence. Nous avons à notre disposition une relation entre la visibilité $V$ des franges et les deux paramètres $\sigma_{z}$ et $\Delta \lambda$. Il est facile de déduire la relation inverse donnant $\sigma_{z}$ en fonction de $V$ et $\Delta \lambda$. La valeur de $\sigma_{z}$ est déduite de l'abaque figure 20. La figure 21 se rapporte à l'étude d'une surface présentant deux valeurs

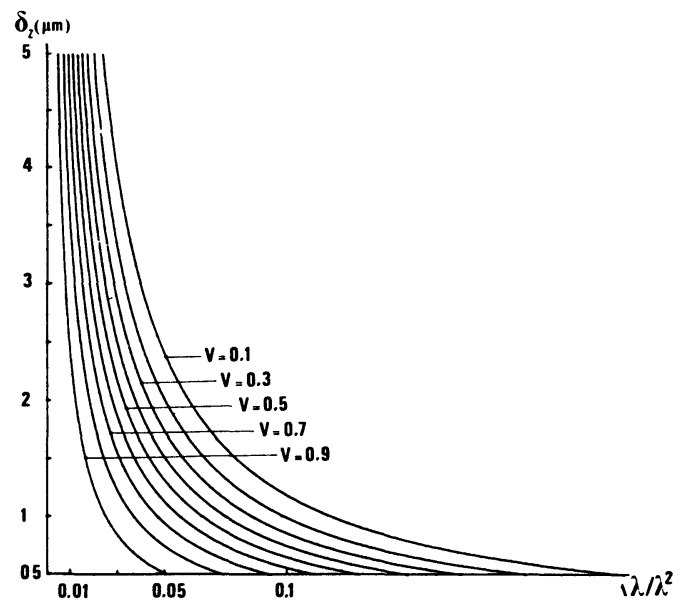

Fig. 20. - Abaque donnant $\sigma_{z}$ en fonction de $\Delta \lambda$ pour différentes valeurs de la visibilité $V$.

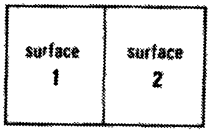

(a)
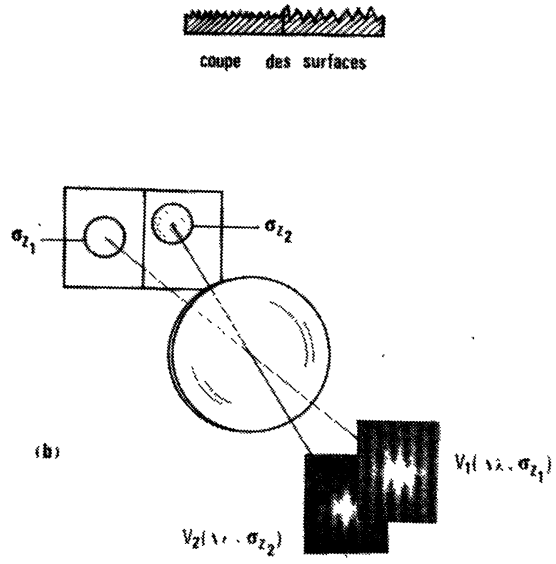

FIG. 21. - Principe de l'étude de rugosités variables en différentes régions de l'échantillon.

moyennes de rugosité. Le processus d'enregistrement reste le même, mais lors de l'exploitation on déplace un diaphragme devant le film photographique. Pour deux expositions successives du diaphragme on relève deux systèmes d'interférence caractérisant les deux rugosités. Cet exemple montre la mise en évidence des variations lentes de la rugosité. 
La précision des mesures est de l'ordre de $5 \%$ pour des rugosités moyennes supérieures à $0,5 \mu \mathrm{m}$. La rapidité d'exécution du test dépend du temps de passage de l'étape d'enregistrement à celle de l'analyse. Dans un banc de mesure en cours de fabrication, le récepteur photographique ne demande qu'un développement à sec, ce qui permet de ramener la durée de la mesure à quelques secondes.

2.2 Coupe TRANSVERSALE D'UNE SURFACE RUGUEUSE PAR HOLOGRAPHIE EN LUMIÈRE POLYCHROMATIQUE. 2.2.1 Enregistrement des hologrammes. - Cette méthode constitue un exemple de ce que nous avons appelé holographie temporelle en lumière polychromatique. Considérons le montage représenté sur la figure 22 où la surface rugueuse analysée, $S$, de profil

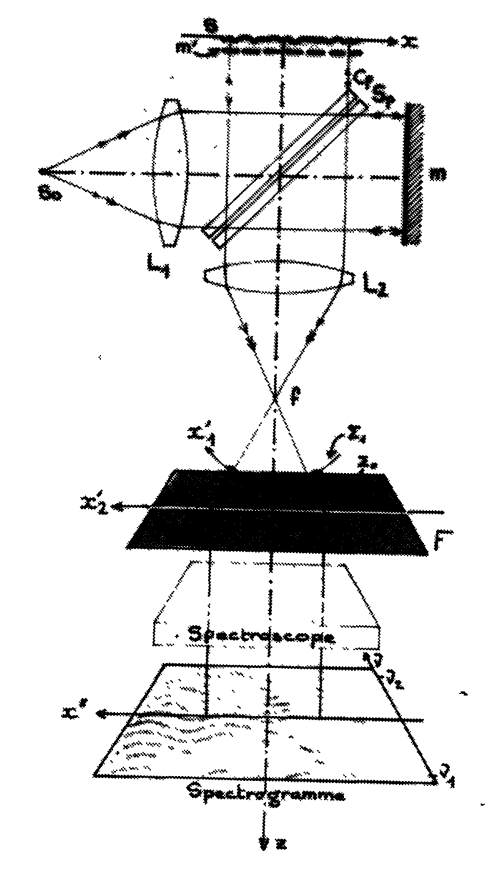

Fig. 22. - Principe de l'enregistrement d'un hologramme temporel de la surface $S$.

$Z(x, y)$ est éclairée par un faisceau de lumière parallèle polychromatique sous incidence normale. Une image de $S$ est projetée sur la fente d'entrée F d'un spectroscope. Le profil de la surface $S$ est supposé fluctuer, par exemple autour du plan $Z=0$. Les surfaces isogènes de la lumière incidente sont planes, celles de la lumière réfléchie fluctuent autour d'un plan. Par exemple par rapport au plan $Z=0$, les fluctuations ont pour amplitude $Z^{\prime}(x, y)=-2 Z(x, y)$. Au plan $Z=0$, correspond au voisinage de la fente d'entrée $\mathrm{F}$ du spectroscope une surface sphérique $\Sigma_{1}$, centrée sur le foyer $\mathrm{F}$ de $\mathrm{L}_{2}$ et autour de laquelle fluctue le profil de l'image de $S$. Sur la figure les points d'abscisses $x, x_{1}^{\prime}, x_{2}^{\prime}$ et $x^{\prime \prime}$ dans les différents plans, sont homologues.
Soit $f(t)$ le profil temporel (ou longitudinal) des perturbations lumineuses incidentes, telles que

$$
f(t) * f(t)=\mathrm{TF}[B(v)],
$$

$B(v)$ étant la densité spectrale de luminance de la source $S$.

Supposons dans un premier temps le miroir $\mathrm{m}$ escamoté. L'échantillon $S$ est réfléchissant. Dans ces conditions, au niveau de la surface $\Sigma_{1}$, les perturbations lumineuses ont pour profil temporel

$$
f\left(t-\frac{Z^{\prime}\left(x^{\prime}, y\right)}{c}\right)=f\left(t+\frac{2 Z(x, y)}{c}\right) .
$$

L'éclairement dans le plan de sortie du spectroscope varie comme

$$
\begin{aligned}
B^{\prime}\left(x^{\prime \prime}, v\right) & =\left|\operatorname{TF}\left[f\left(t-\frac{Z^{\prime}\left(x^{\prime}, 0\right)}{c}\right)\right]\right|^{2} \\
& =\left|\exp \left(-2 \pi j v \frac{Z^{\prime}}{c}\right) \operatorname{TF}[f(t)]\right|^{2} \\
& =|\operatorname{TF}[f(t)]|^{2}=B(v) .
\end{aligned}
$$

Le retard $Z^{\prime}(x, 0)$ se traduit au niveau du spectre par la présence d'un terme de phase, $\mathrm{e}^{-j 2 \pi v Z^{\prime} / c}$, qui échappe à l'observation classique à l'aide des récepteurs quadratiques. Ici, le spectre visible n'est donc pas modifié par la réflexion sur $S$; et les fluctuations locales de $Z^{\prime}(x, y)$ sont inaccessibles par cette voie.

Replaçons le miroir $\mathrm{m}$. A la surface plane réfléchiesante de ce miroir, correspond une surface sphériqus image $\Sigma_{0}$ au voisinage de $\mathrm{F}$, la surface $\Sigma_{0}$ sert alors de surface de référence pour $\Sigma_{1}$. Soit $Z=Z_{0}$ la position de l'image $\mathrm{m}^{\prime}$ de $\mathrm{m}$ à travers la séparatrice et $Z_{0}^{\prime}=-2 Z_{0}$ la distance correspondante entre les surfaces $\Sigma_{0}$ et $\Sigma_{1}$. La réponse temporelle du système s'écrit maintenant :

$$
\begin{aligned}
f\left[t-\frac{Z^{\prime}\left(x^{\prime}, y\right)}{c}\right]+f\left[t-\frac{Z_{0}^{\prime}}{c}\right]= \\
=f\left(t^{\prime}\right) \otimes\left[\delta\left(t^{\prime}-\frac{Z^{\prime}\left(x^{\prime}, y\right)}{c}\right)+\delta\left(t^{\prime}-\frac{Z_{0}^{\prime}}{c}\right)\right] \\
=f\left(t^{\prime}\right) \otimes h\left(t^{\prime}\right),
\end{aligned}
$$

$\delta\left(t^{\prime}\right)$ étant une mesure de Dirac. Le terme $h\left(t^{\prime}\right)$ est la réponse impulsionnelle temporelle du système. La répartition d'éclairement à la sortie du spectroscope est $B^{\prime \prime}\left(x^{\prime \prime}, v\right) \propto\left|\operatorname{TF}\left[f\left(t^{\prime}\right) \otimes h(t)\right]\right|^{2} ;$ soit

$$
B^{\prime \prime}\left(x^{\prime \prime}, v\right) \propto B(v) \cdot\left\{1+\cos 2 \pi \frac{v}{c} 2\left[Z(x, y)-Z_{0}\right]\right\} .
$$

On voit qu'en faisant suivre ou précéder les surfaces isogènes caractérisant l'objet par une surface isogène sphérique de référence, on obtient un spectrogramme présentant des cannelures (Fig. 23) (certaines longueurs d'onde manquent), dont la forme et le nombre dans 


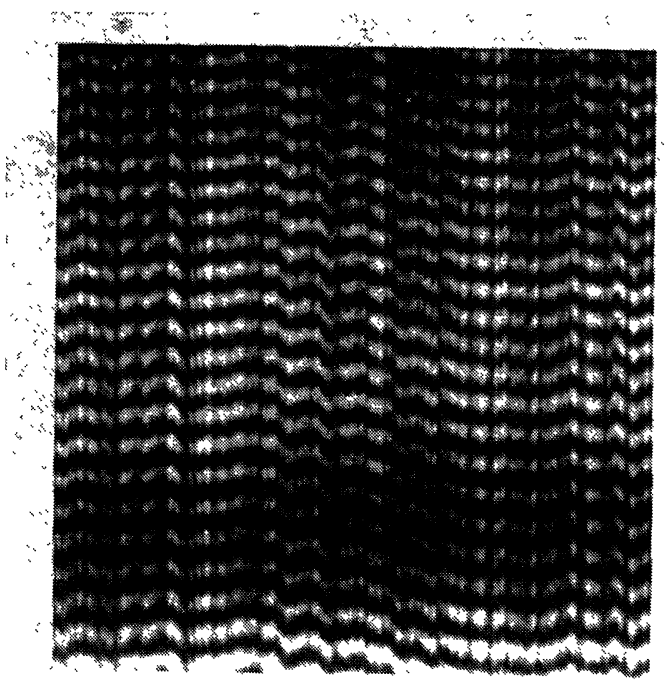

FIG. 23. - Hologramme « temporel » d'une surface rugueuse. (Explication dans le texte.)

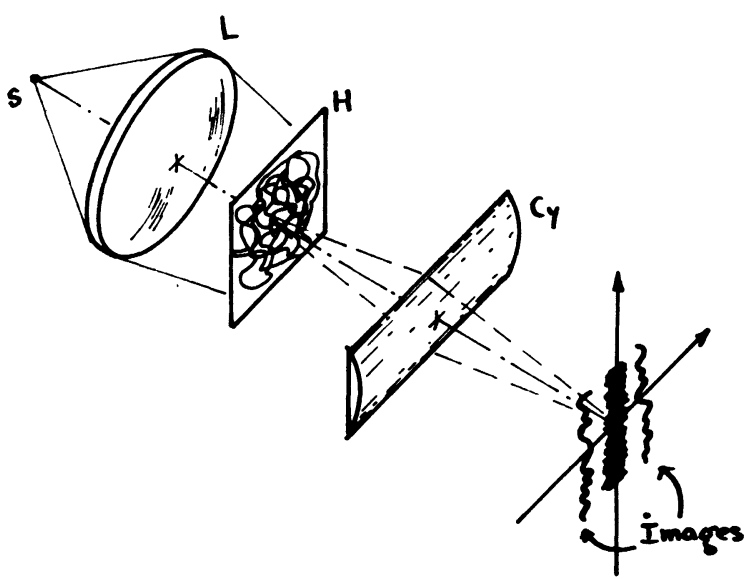

FIG. 24. - Restitution du profil de la surface par diffraction d'une onde monochromatique à travers l'hologramme $\mathrm{H}$. La lentille cylindrique $\mathrm{C}_{y}$ conjugue le plan de l'hologramme et celui des images. (Fig. 25.)

intervalle de fréquences temporelles donné $\Delta v$ dépendent du profil de la surface $S$ :

$$
N=\frac{2 \Delta v}{c}\left[Z(x, y)-Z_{0}\right] .
$$

Le spectrogramme permet donc d'évaluer aisément $Z(x, y)$ en comptant $N$. Mais nous allons montrer que plus simplement encore, il constitue un véritable hologramme des variations de $Z(x)$.

2.2.2 Production d'une image du profil $Z(x)$ de l'échantillon rugueux. - Si l'on travaille dans une
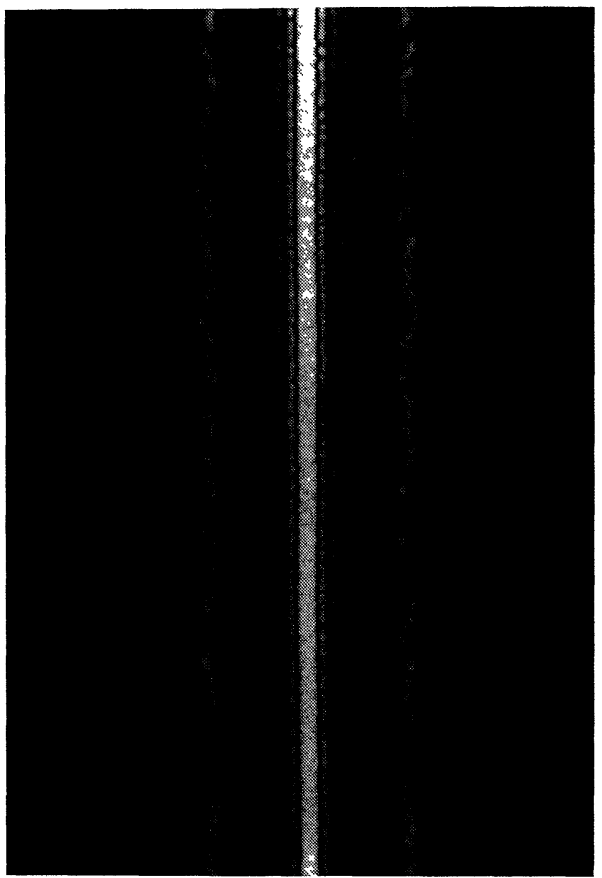

Fig. 25. - Images anamorphosées du profil d'une surface. Cette image représente une amplification des défauts de la surface étudiée (ici, $1 \mu \mathrm{m}$ environ). La largeur de l'image correspond à environ $1 \mathrm{~cm}$ sur la surface.

bande spectrale $\Delta v=v_{2}-v_{1}$ étroite (dispersion quasi linéaire), ou si l'on utilise un spectroscope particulier, à double dispersion, l'éclairement dans le spectrogramme a pour expression :

$$
E\left(x^{\prime \prime}, \eta\right)=B(k \eta)\left[1+\cos 2 \pi k \eta \frac{2 Z(x)-2 Z_{0}}{c}\right],
$$

O $\eta$ étant un axe géométrique parallèle à l'axe des fréquences temporelles sur le spectrogramme. Si cette répartition est enregistrée sur une émulsion photographique dans des conditions de linéarité entre éclairement et transmittance complexe après développement, $t\left(x^{\prime \prime}, \eta\right)$, il vient :

$$
\begin{aligned}
t\left(x^{\prime \prime}, \eta\right) \propto E\left(x^{\prime \prime}, \eta\right) & = \\
& =B(k \eta) \cdot\left[1+\cos 2 \pi k \eta \frac{2 Z(x)-2 Z_{0}}{c}\right] .
\end{aligned}
$$

Formons la figure de diffraction à l'infini de ce cliché, dans un plan $X \mathrm{O}^{\prime} Y\left(\mathrm{O}^{\prime} Y\right.$ parallèle à $\left.\mathrm{O} \eta\right)$. Formons également son image suivant $\mathrm{O}^{\prime} X$ (parallèle à $O x^{\prime \prime}$ ). Ceci est réalisé en optique sphéro-cylindrique (Fig. 24). L'amplitude dans le plan $X \mathrm{O}^{\prime} Y$ s'écrit

$$
\begin{gathered}
A(X, Y) \propto \int_{-\infty}^{+\infty} B(k \eta)\left[1+\cos 2 \pi k \eta \frac{2 Z(x, y)-2 Z_{0}}{c}\right] \mathrm{e}^{-j 2 \pi v_{0} k^{\prime} Y \eta / c} \mathrm{~d} \eta \propto \operatorname{TF}[B(k \eta)] \otimes \\
\otimes \mathrm{TF}\left[1+\cos 2 \pi k \eta \frac{2 Z\left(x^{\prime}\right)-2 Z_{0}}{c}\right]=\left[f\left(\frac{k^{\prime} \sigma_{0} Y^{\prime}}{k}\right) * f\left(\frac{k^{\prime} \sigma_{0} Y^{\prime}}{k}\right)\right] \otimes \\
\otimes\left\{\delta\left(Y^{\prime}\right)+\frac{1}{2} \delta\left[Y^{\prime}-\frac{2 k}{k^{\prime} v_{0}}\left(Z\left(x^{\prime}\right)-Z_{0}\right)\right]+\frac{1}{2} \delta\left[Y^{\prime}+\frac{2 k}{k^{\prime} v_{0}}\left(Z\left(x^{\prime}\right)-Z_{0}\right)\right]\right\} .
\end{gathered}
$$


On voit que les termes

et

$$
\frac{1}{2} \delta\left[Y^{\prime}-\frac{2 k}{k^{\prime} v_{0}}\left(Z\left(x^{\prime}\right)-Z_{0}\right)\right]
$$

$$
\frac{1}{2} \delta\left[Y^{\prime}+\frac{2 k}{k^{\prime} v_{0}}\left(Z\left(x^{\prime}\right)-Z_{0}\right)\right]
$$

correspondent à deux images du profil $Z\left(x^{\prime}\right)$ : on observe deux courbes symétriques dont un point d'abscisse $X=p x^{\prime \prime}$ ( $p$, facteur dépendant de la géométrie du dispositif) a pour ordonnée $Y=\left(K\left(Z^{\prime} x^{\prime}\right)-Z_{0}\right)$, $Z_{0}$ étant une constante arbitraire (Fig. 25). 